\title{
NIRS Measurements with Elite Speed Skaters: Comparison between the Ice Rink and the Laboratory
}

\author{
Catherine Hesford $^{1,2}$, Marco Cardinale ${ }^{2}$, Stewart Laing ${ }^{2}$ \& Chris E. Cooper $^{1}$ \\ ${ }^{1}$ School of Biological Sciences, University of Essex, Colchester, UK \& ${ }^{2}$ British Olympic \\ Medical Institute, University College London Hospital, UK
}

\begin{abstract}
Wearable, wireless near-infrared (NIR) spectrometers were used to compare changes in on-ice short-track skating race simulations over $1500 \mathrm{~m}$ with a three-minute cycle ergometry test at constant power output $(400 \mathrm{~W})$. The subjects were six male elite short-track speed skaters. Both protocols elicited a rapid desaturation $(\Delta \mathrm{TSI} \%)$ in the muscle during early stages (initial $20 \mathrm{~s}$ ), however asymmetry between right and left legs was seen in $\Delta \mathrm{TSI} \%$ for the skating protocol, but not for cycling. Individual differences between skaters were present in both protocols. Notably, one individual who showed a relatively small TSI\% change $(-10.7 \%$, group mean $=-26.1 \%$ ), showed a similarly small change during the cycling protocol $(-5.8 \%$, group mean $=-14.3 \%)$. We conclude that NIRS-detected leg asymmetry is due to the specific demands of short-track speed skating. However, heterogeneity between individuals is not specific to the mode of exercise. Whether this is a result of genuine differences in physiology or a reflection of differences in the optical properties of the leg remains to be determined.
\end{abstract}

\section{Introduction}

Near-infrared spectroscopy (NIRS) has been used to investigate exercise-specific oxygen metabolism and hemodynamics in the laboratory for a range of sports including: cycling [1, 2]; sailing [3]; and speed skating [4]; for review see [5]. However, the recent development of wearable wireless, portable NIR spectrometers has enabled the investigation of changes in local muscle metabolism and blood flow during exercise in a more realistic sporting setting.

In short-track speed skating, the combination of high intramuscular forces, long duty cycle of the gliding phase in the skating motion, and the low sitting position adopted leads to a restriction of blood flow to the working muscle: the 'reduced blood flow hypothesis' [6]. Previous research using NIR techniques has centered on laboratory testing using treadmill skating, and has shown that the low sitting position of the skater elicits a lower submaximal $\mathrm{VO}_{2}$ [7], increased blood lactate concentration [8], and increased quadriceps deoxygenation [4] when compared to 
upright skating. On-ice tests have shown that skating at higher speeds produces a greater deoxygenation in the quadriceps muscle [8]. The current study utilized a wearable, wireless NIR spectrometer to measure the local muscle oxygenation and blood volume changes during an on-ice race simulation, enabling investigation of the asymmetric effects on right and left legs of skating around a short-track oval. A separate laboratory cycle test with the same subjects was used to confirm whether any asymmetry in NIRS measurements was specific to the technical and biomechanical requirements of the event.

\section{Methods}

Six elite male short-track speed skaters (mean \pm SD age; $23 \pm 1.8$ years, height; $1.8 \pm 0.1 \mathrm{~m}$, mass; $80.1 \pm 5.7 \mathrm{~kg}$, mid-thigh skin fold thickness; $6.8 \pm 2.2 \mathrm{~mm}$ ) of Olympic standard, took part in this study. All subjects gave their written informed consent prior to participation. The study was approved by the ethics committee of University College London.

Protocol 1 involved each subject completing a $1500 \mathrm{~m}$ on-ice race simulation. Testing took place on a short-track speed skating oval $(111.12 \mathrm{~m})$ approved for international competition. Before completing race simulation, subjects were informed to undertake their own warm-up, as they would when preparing for a competitive race. Race simulation TTs were completed individually and subjects were informed to try and achieve the fastest time possible. Protocol 2 was a laboratorybased task which involved 3 minutes cycling at a constant cadence (90rpm) and power output $(400 \mathrm{~W})$ on an electro-magnetically braked cycle ergometer (Excalibur Sport V2, Lode, the Netherlands). This was immediately preceded by a 5minute warm-up at a constant load (90W) and constant cadence (90rpm). Protocol 2 took place 4 weeks after protocol 1 .

In both protocols, muscle oxygenation in both left and right vastus lateralis was continuously monitored using a recently developed wireless, dual-wavelength Spatially Resolved Spectrometer (Portamon, Artinis Medical Systems, BV, the Netherlands). The unit is self-contained and compact, measuring $83 \times 52 \times 20 \mathrm{~mm}$ and weighing $84 \mathrm{~g}$, including battery. It houses three pairs of light emitting diodes (LEDs) which emit light of wavelengths 760 and $850 \mathrm{~nm}$, and are positioned $30 \mathrm{~mm}, 35 \mathrm{~mm}$, and $40 \mathrm{~mm}$ from the detector. This device has previously been used to investigate muscle oxygenation and hemodynamics in both a controlled laboratory environment [9], and in a field setting: thus utilizing the portable, wireless nature of the instrument [10-12]. The devices were positioned on the belly of the vastus lateralis muscle, midway between the greater trochanter of the femur and the lateral epicondyle. To ensure the optodes and detector did not move relative to the subject's skin, the device was fixed into position using surgical tape, and then secured with a bandage, which was wound around the thigh a number of times. The same attachment procedure was undertaken for both protocols. Changes in 
optical density were converted to changes in oxygenation $\left(\Delta \mathrm{Hb}_{\mathrm{diff}}, \Delta \mathrm{HbO}_{2}-\right.$ $\Delta \mathrm{HHb})$, blood volume $\left(\Delta \mathrm{tHb}, \Delta \mathrm{HbO}_{2}-\Delta \mathrm{HHb}\right)$ and an SRS-derived measure of tissue oxygen saturation (\%TSI).

\section{Results}

The $1500 \mathrm{~m}$ race simulation was completed in $151.03 \pm 4.04 \mathrm{~s}$. At exercise onset in both on-ice skating and laboratory cycling protocols, there was a rapid decrease in total hemoglobin $(\mathrm{tHb})$, followed by a steady increase throughout the remainder of the exercise. Both protocols also elicited a rapid desaturation $\left(\Delta \mathrm{Hb}_{\mathrm{diff}}, \Delta \mathrm{TSI} \%\right)$ in the muscle during early stages (initial 20s). The maximum decrease in saturation $\left(\Delta \mathrm{TSI}_{\max }\right)$ for the skating test was $\Delta 26.1 \%$, and for the cycling test was $\Delta 14.3 \%$.

By examining the group average data for both protocols (Fig. 1), it can be seen that a pattern of asymmetry emerged during the skating race simulation: the right leg TSI remained desaturated, but the left leg TSI gradually increased with time. This asymmetry in skating can also be seen from the observed changes in $\mathrm{Hb}_{\text {diff }}$ during the race simulation, but there is no apparent asymmetry in the $\mathrm{tHb}$ values. However, there is no such pattern in any of the measured parameters during the cycling protocol, in which left and right leg changes are closely matched in magnitude throughout the exercise.

Figure 2 presents the $\Delta \mathrm{tHb}$ values for each individual during both protocols. During the skating protocol, it was noted that the majority of skaters showed an initial reduction in $\mathrm{tHb}$ at race start (presumably due to a blood squeezing effect of muscle contraction at exercise onset), followed by a gradual increase in $\mathrm{tHb}$ throughout the remainder of the exercise, presumably as a consequence of a blood flow increase. In most cases, this results in a final tHb significantly higher than the baseline value. However, one subject (C) showed very little change in tHb during the race, and his final values in both legs were notably lower than most. The cycling protocol produced a more pronounced difference between the tHb changes of this individual and the rest of the group: Fig 2 shows that during cycling, $\mathrm{tHb}$ did not increase in either leg of subject $\mathrm{C}$ following the initial decrease at exercise onset. This is in contrast to all other subjects. Subject $C$ also showed the smallest desaturation in both skating and cycling (results not shown).

\section{Conclusions}

The asymmetry observed between the right and left leg during the skating protocol is most likely due to the specific demands of this sport. Skaters travel around the track in an anti-clockwise direction, which means the right leg is always the 'outside' leg. When travelling around the corners at the highest speeds, the skater tra- 
vels solely on the right leg, which is in a sustained isometric contraction, whilst the left leg is free of the ice. This technique is called the 'hang phase'. During this technique, the left leg is able to resaturate, whereas the right leg cannot [13]. We have previously suggested from $500 \mathrm{~m}$ studies that the cumulative effect of these "hang" phases could lead to asymmetry in oxygenation and blood volume that persisted throughout the simulation [13]. This study confirmed this effect for the $1500 \mathrm{~m}$ race simulation. The laboratory cycling protocol demonstrated that the asymmetry was due to the specific demands of skating, rather than being due to an intrinsic difference between the right and left quadriceps muscles of this particular subject group.

Individual differences in NIRS-detected parameters were replicated in the sports-specific and laboratory protocols, notably the atypical tHb changes in subject $\mathrm{C}$, which were present in both skating and cycling. It is not possible to tell whether these inter-individual variations are due to specific physiological differences or variability in light transport through the leg. However, it is clear that they do not relate to the specifics of the biomechanical performance in the sports event.

This study highlights the potential benefits of using portable NIRS in realistic sporting settings to gain rapid, real-time information pertaining to muscle oxygenation changes and hemodynamics during exercise. This study also illustrates the importance of comparing controlled laboratory and field studies in the same individuals before using NIRS as a tool for individualised sports-specific advice.

\section{References}

1. Takaishi, T., K. Ishida, K. Katayama, et al., (2002) Effect of cycling experience and pedal cadence on the near-infrared spectroscopy parameters. Med Sci Sports Exerc, 34(12): 20622071

2. Racinais, S., D. Bishop, R. Denis, et al., (2007) Muscle deoxygenation and neural drive to the muscle during repeated sprint cycling. Med Sci Sports Exerc, 39(2): 268-274

3. Vogiatzis, I., D. Tzineris, D. Athanasopoulos, et al., (2008) Quadriceps oxygenation during isometric exercise in sailing. Int J Sports Med, 29(1): 11-15

4. Rundell, K.W., S. Nioka, and B. Chance, (1997) Hemoglobin/myoglobin desaturation during speed skating. Med Sci Sports Exerc, 29(2): 248-258

5. Hamaoka, T., K.K. McCully, V. Quaresima, et al., (2007) Near-infrared spectroscopy/imaging for monitoring muscle oxygenation and oxidative metabolism in healthy and diseased humans. J Biomed Opt, 12(6): 16

6. Snyder, A.a.F., C., Physiology and Nutrition for Skating, in Perspectives in Exercise Science and Sports Medicine, H.G.K. D. R Lamb, and R. Murray, Editor. 1994, Cooper Publishing Group: Carmel, IN p. 181-219.

7. Rundell, K.W., (1996) Compromised oxygen uptake in speed skaters during treadmill in-line skating. Med Sci Sports Exerc, 28(1): 120-127

8. Foster, C., K.W. Rundell, A.C. Snyder, et al., (1999) Evidence for restricted muscle blood flow during speed skating. Med Sci Sports Exerc, 31(10): 1433-1440

9. Shadgan, B., W.D. Reid, R. Gharakhanlou, et al., (2009) Wireless near-infrared spectroscopy of skeletal muscle oxygenation and hemodynamics during exercise and ischemia. Spectrosc Int J, 23(5-6): 233-241 
10. Buchheit, M., P.B. Laursen, and S. Ahmaidi, (2009) Effect of prior exercise on pulmonary $\mathrm{O} 2$ uptake and estimated muscle capillary blood flow kinetics during moderate-intensity field running in men. J Appl Physiol, 107(2): 460-470

11. Buchheit, M., D. Bishop, B. Haydar, et al., (2010) Physiological Responses to Shuttle Repeated-Sprint Running. Int J Sports Med, 31(6): 402-409

12. Buchheit, M., (2010) Performance and physiological responses to repeated-sprint and jump sequences. Eur J Appl Physiol, 110(5): 1007-1018

13. Hesford, C., Laing, SJ., Cardinale, M., Cooper, CE, (2011) Asymmetry of quadriceps muscle oxygenation during elite short-track speed skating. Med Sci Sports Exerc, In Press
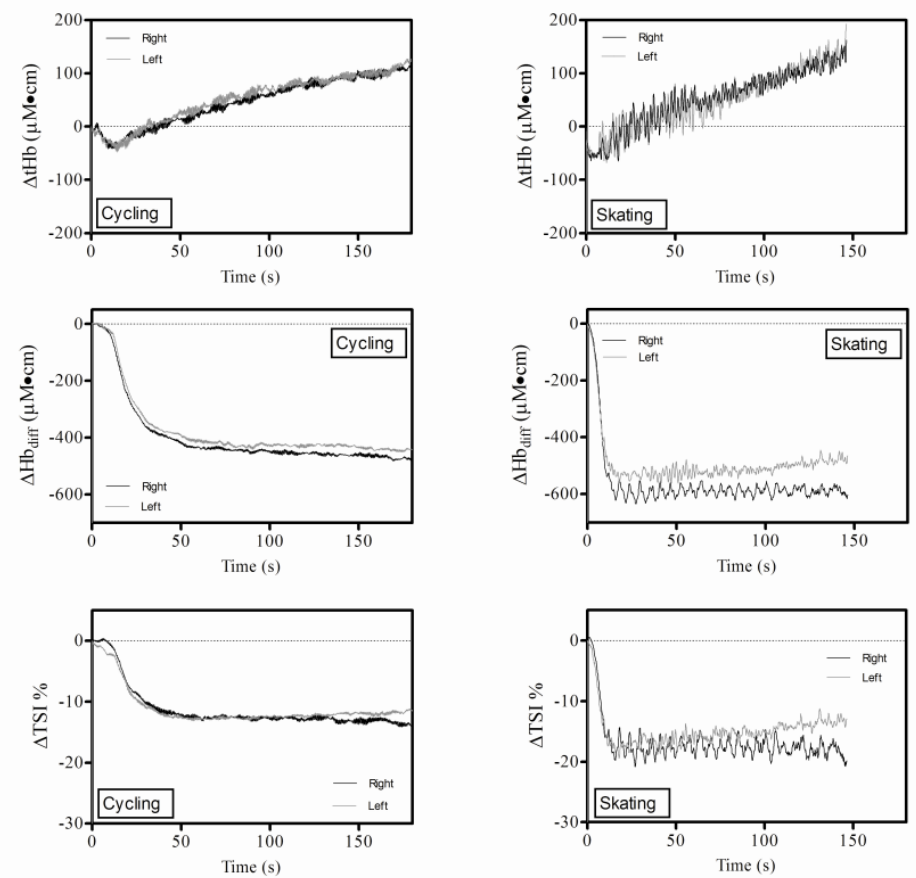

Fig. 1. Mean changes from baseline in 3 NIRS-detected parameters ( $\left.\mathrm{tHb} ; \mathrm{Hb}_{\mathrm{diff}} ; \mathrm{TSI} \%\right)$ in the right and left VL during both skating and cycling protocols. Following initial desaturation at exercise onset, asymmetry between right and left leg $\mathrm{Hb}_{\text {diff }}$ and TSI data can be seen during skating, but not during the cycling protocol. 

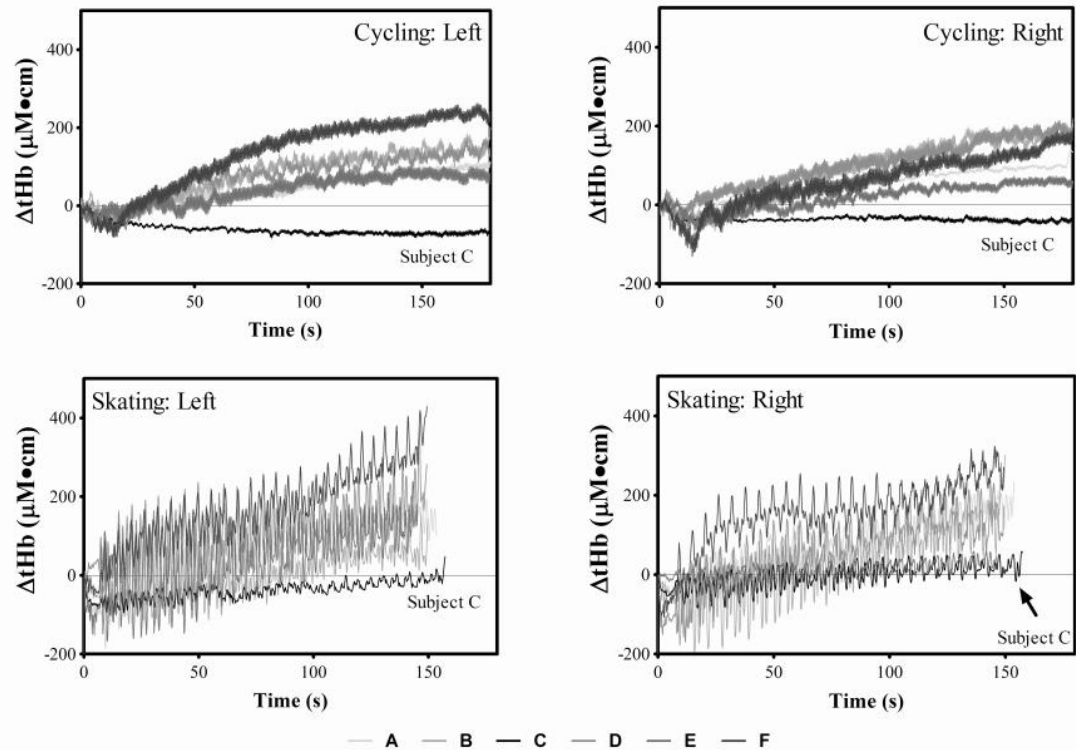

Fig. 2. tHb changes in both right and left VL of each subject during both cycling and skating protocols. The high frequency variations seen in skating are due to changes in local blood volume due to different body positions at specific stages of the lap (e.g. straight, corners). This has previously been described [13]. 